\title{
ALAVANCAGEM FINANCEIRA E RENTABILIDADE NAS EMPRESAS BRASILEIRAS LISTADAS NA B3
}

\section{FINANCIAL LEVERAGE AND PROFITABILITY IN BRAZILIAN COMPANIES LISTED ON THE B3}

\section{APALANCAMIENTO FINANCIERO Y RENTABILIDAD EN LAS EMPRESAS BRASILEÑAS LISTADAS EM LA B3}

Recebido em: 28/01/2018

Avaliado em: 19/07/2019

Reformulado em:04/08/2019

Aceito para publicação em: 14/11/2019

Publicado em: 30/11/2019

Editor Responsável: Tarcísio Pedro da Silva

\author{
Paulo Fernando Marschner ${ }^{1}$ \\ Vanessa Rabelo Dutra ${ }^{2}$ \\ Paulo Sergio Ceretta ${ }^{3}$
}

\section{RESUMO}

Este artigo analisa a relação entre a alavancagem e a rentabilidade nas empresas brasileiras não financeiras de capital aberto no período de 2010 a 2015. Para atingir o objetivo proposto foram analisadas 75 empresas listadas na B3 S.A - Brasil, Bolsa, Balcão, a partir de dados coletados no banco de dados Economática. O procedimento metodológico empregado foi o método de análise de dados em painel com threshold proposto por Hansen (1999). No modelo foi considerada como variável dependente a rentabilidade, sendo explicada pela alavancagem financeira, e controladas pelas variáveis tamanho e crescimento. Seguindo a proposição de Fattouh, Harris e Scaramozzino (2008), a variável "Alavancagem" é definida como o threshold do modelo, e após estimado, foram encontrados dois regimes balizados por esta variável, que possibilitou a segmentação da amostra em empresas com maior e menor alavancagem. As evidências empíricas, sugerem que no primeiro regime, onde estão as empresas com menor alavancagem, não existe uma relação significativa entre alavancagem financeira e rentabilidade, no segundo, por sua vez, onde estão as empresas com maior alavancagem, existe um efeito negativo da alavancagem sobre a rentabilidade. A variável tamanho é significativa e positiva em ambos os regimes, porém o crescimento não foi significativo em nenhum regime. As conclusões gerais deste estudo são favoráveis a Teoria de Pecking Order, e sugerem a existência de uma relação não linear entre alavancagem financeira e rentabilidade nas empresas brasileiras de capital aberto. Esse estudo amplia o escopo dos exames empíricos sobre rentabilidade e alavancagem a partir de uma metodologia que proporciona uma forma diferenciada de analisar os resultados a fim de obter um relacionamento estrutural específico entre as variáveis.

Palavras-chave: Alavancagem Financeira. Rentabilidade. Threshold.

\footnotetext{
1 Mestrando em Administração pela Universidade Federal de Santa Maria (UFSM); E-mail: paulofernandomarschner@hotmail.com

${ }_{2}^{2}$ Doutoranda em Administração pela Universidade Federal de Santa Maria (UFSM); E-mail: vanessard@gmail.com

${ }^{3}$ Doutor em Engenharia de Produção pela Universidade Federal de Santa Catarina (UFSC); Professor do Programa de Pós-Graduação em Administração da Universidade Federal de Santa Maria (UFSM); E-mail: ceretta10@gmail.com
} 


\begin{abstract}
This article analyzes the relationship between financial leverage and profitability in Brazilian nonfinancial companies in the period from 2010 to 2015. In order to reach the proposed objective, 75 companies listed in B3 S.A - Brazil, Bolsa, Balcão, were analyzed from data collected in the Economática database. The methodological procedure used was the panel data analysis method with threshold proposed by Hansen (1999). In the model the profitability was considered as a dependent variable, being explained by financial leverage, and controlled by size and growth variables. Following the proposition of Fattouh, Harris and Scaramozzino (2008), the variable "Leverage" is defined as the threshold of the model, and after estimation, two regimes were entered into by this variable, which allowed the segmentation of the sample in companies with higher and lower leverage. The empirical evidence suggests that in the first regime, where the companies with the lowest leverage are, there is no significant relationship between financial leverage and profitability. In the second, in turn, where the companies with the highest leverage are, there is a negative effect of the financial leverage on the profitability. The variable size is significant and positive in both regimens, but growth was not significant in any regimen. The general conclusions of this study are favorable to the Pecking Order Theory and suggest the existence of a nonlinear relationship between financial leverage and profitability in Brazilian publicly traded companies. This study expands the scope of profitability and leverage empirical examinations from the methodology that provides a differentiated way to analyze results to obtain a specific relationship between variables.
\end{abstract}

Keywords: Financial Leverage. Profitability. Threshold.

\title{
RESUMEN
}

Este artículo analiza la relación entre el apalancamiento financiero y la rentabilidad en las empresas brasileñas no financieras de capital abierto en el período de 2010 a 2015. Para alcanzar el objetivo propuesto se analizaron 75 empresas listadas en B3 S.A - Brasil, Bolsa, Balcão, a partir de los datos recopilados en la base de datos de Economática. El procedimiento metodológico empleado fue el método de análisis de datos en panel con threshold propuesto por Hansen (1999). En el modelo fue considerada como variable dependiente la rentabilidad, siendo explicada por el apalancamiento financiero, y controladas por las variables tamaño y crecimiento. La variable "apalancamiento" se define como el threshold del modelo, y después de estimado, se introdujeron dos regímenes balizados por esta variable, que posibilitó la segmentación de la muestra en empresas con mayor y mayor, menor apalancamiento. Las evidencias empíricas sugieren que en el primer régimen, donde están las empresas con menor apalancamiento, no existe una relación significativa entre apalancamiento financiero y rentabilidad, en el segundo, a su vez, donde están las empresas con mayor apalancamiento, existe un efecto negativo del apalancamiento financiera sobre la rentabilidad. La variable tamaño es significativa y positiva en ambos regímenes, pero el crecimiento no fue significativo en ningún régimen. Las conclusiones generales de este estudio son favorables a la Teoría de Pecking Order, y sugieren la existencia de una relación no lineal entre apalancamiento financiero y rentabilidad en las empresas brasileñas de capital abierto. Este estudio amplía el alcance de los exámenes empíricos a cerca de la rentabilidad y del apalancamiento financiero a través de una metodología que proporciona una forma diferenciada de analizar los resultados para obtener una relación estructural específica entre las variables.

Palabras clave: Apalancamiento financiero. Rentabilidad. Threshold.

\section{INTRODUÇÃO}

Um assunto recorrente na Teoria Financeira tem sido a forma como uma empresa é financiada, ou seja, sua estrutura de capital, e como essa afeta o valor da firma, sua rentabilidade e sua política 
de investimentos (Lang, Okef, \& Stulz, 1996). O uso do capital de terceiros na estrutura de capital das empresas é pertinente devido ao efeito da alavancagem financeira, que se refere à possibilidade de ganhos superiores para os proprietários de uma determinada empresa, com o uso do endividamento. Em vista disto, Titman e Wessels (1988) sugerem que empresas com baixa rentabilidade possam financiar suas despesas com um aumento do uso do capital de terceiros.

Em se tratando de mercados perfeitos, Modigliani e Miller (1958) sugerem que o valor de mercado de uma empresa não é determinado pela sua estrutura de capital, mas sim, pela composição de seus ativos. Trata-se de um modelo com vários pressupostos, inconsistentes com o contexto atual e da época, no qual mercados perfeitos são aqueles em que não há custos, impostos, assimetria de informações, e onde a dívida de uma empresa é livre de risco. Essa proposição inicial foi posteriormente revisada pelos autores, que incorporaram os benefícios fiscais do endividamento (Modigliani, \& Miller, 1963). Considerando a partir de então, que o custo do capital de terceiros seria menor em relação aos recursos próprios. Foi a partir da combinação destes fatores, que se estabeleceu a Teoria de Trade-off, a qual sugere que a estrutura de capital ótima de uma empresa seria determinada pelo equilíbrio entre os benefícios fiscais da dívida e os custos de falência associados à alavancagem (Myers, 1984).

No entanto, de acordo com Myers (1977), a Teoria Financeira deve ser capaz de explicar por que os benefícios fiscais da dívida não levam as empresas a usarem o máximo possível de capital de terceiros, bem como, explicar porque algumas empresas contratam mais recursos do que outras. Em meio a estas interlocuções e divergências, outra teoria foi desenvolvida no campo da estrutura de capital, denominada Pecking Order, ou da ordem de preferência, que leva em consideração a assimetria de informações existente no mercado de capitais (Myers, \& Majluf,1984).

A Teoria de Pecking Order, sugere que não existe um nível ótimo de endividamento, já que a escolha de capital das empresas é resultado das suas necessidades de financiamento. Esta teoria propõe que as empresas seguem uma ordem hierarquia de fontes de financiamento, onde, primeiramente, seriam utilizados os recursos gerados de forma interna. Em seguida, seriam utilizados recursos de terceiros, via endividamento, e a última opção seria a emissão de novas ações. Ou seja, as empresas aumentariam o financiamento externo apenas quando os recursos internos não tenham sido suficientes (Myers, 1984; Myers, \& Majluf, 1984).

Posterior a estes, diversos trabalhos de cunho teórico e empírico foram desenvolvidos tentando explicar os fatores que podem determinar a estrutura de capital. Bradley, Jarrell e Kim (1984) por exemplo, desenvolveram um modelo que sintetiza a teoria de estrutura de capital ótima, incorporando impostos pessoais sobre o patrimônio líquido e sobre a renda das obrigações, custos esperados de dificuldades financeiras (custos de falência e custos de agência) e escudos fiscais positivos não relacionados à dívida. Harris e Raviv (1991) por sua vez, desenvolvem uma revisão abrangente sobre as teorias da estrutura de capital com base nos custos de agência, informações assimétricas, interações de mercado e controle corporativo.

Além disso, já foram documentadas evidências de que em nível agregado, a alavancagem das empresas é semelhante em todos os países do G7 (Rajan, \& Zingales, 1995), ou ainda, que as decisões de estrutura de capital de empresas de países em desenvolvimento são afetadas pelas mesmas variáveis que afetam os países desenvolvidos (Booth et al., 2001). Ou seja, embora alguns dos insights sejam portáveis entre os países, as diferenças que afetam as escolhas de capital tendem a ser causadas por fatores específicos ou características institucionais em funcionamento em cada país (Booth et al., 2001).

Dentre as descobertas importantes mais recentes, pode-se destacar as várias influências diretas e indiretas dos níveis de tempo, firma, indústria e país sobre os determinantes da alavancagem das empresas, bem como as diferenças estruturais no comportamento financeiro entre empresas de países desenvolvidos e emergentes (Kayo, \& Kimura, 2011). Outra evidência relevante trata da estabilidade da estrutura de capital (Deangelo, \& Roll, 2015). Embora os trabalhos que versam sobre a estrutura de capital tenham sido em grande maioria conduzidos em países com mercados desenvolvidos, as 
questões discutidas internacionalmente também foram abordadas por diversos estudos brasileiros (Famá, \& Kayo, 1997; Perobelli, \& Famá, 2003; Bastos, Nakamura, \& Basso, 2009; Ceretta et al., 2009; Bastos, \& Nakamura, 2009; Corrêa, Basso, \& Nakamura, 2013; Silva et al., 2016).

Em virtude disso, alguns fatos estilizados acerca das decisões de estrutura de capital foram investigados na literatura. Em suma, a decisão de alavancagem de uma empresa estaria ligada à sua lucratividade, capacidade de fornecer garantias, tamanho e crescimento. Nesse sentido, uma questão de considerável debate têm sido a relação entre alavancagem financeira e rentabilidade. Apesar do grande número de pesquisas, os trabalhos anteriores indicaram uma falta de consenso sobre a relação entre estas variáveis. Além disso, estes trabalhos presumem que a relação entre alavancagem e rentabilidade é linear, quando na verdade existem evidências de que essa relação tende a ser não linear (Cheng, Liu, \& Chien, 2010; Cuong, \& Canh, 2012; Lin, \& Chang, 2015; Jaisinghani, \& Kanjilal, 2017), ou seja, que o efeito de uma variável sobre outra poderá ser diferente de acordo com a posição da empresa na distribuição de alavancagem (Fattouh, Harris, \& Scaramozzino, 2008).

Com foco em tal perspectiva o objetivo deste trabalho é analisar a relação não linear entre alavancagem financeira e rentabilidade nas empresas brasileiras listadas na B3 S.A - Brasil, Bolsa, Balcão no período de 2010 a 2015. Para atingir o objetivo proposto a amostra original foi dividida em duas subamostras, com maior e menor alavancagem e posteriormente verificado o impacto da alavancagem sobre a rentabilidade destas empresas. Esse procedimento foi estimado por meio da abordagem metodológica desenvolvida por Hansen (1999).

O presente estudo amplia a literatura contábil e de finanças, examinando empiricamente o relacionamento não linear entre alavancagem financeira e rentabilidade em empresas brasileiras. Dessa forma, este trabalho proporciona uma extensão ao conhecimento existente porque adota um método mais robusto que pode oferecer uma alternativa de análise diferenciada. Portanto, tem-se a pretensão de aplicar testes econométricos que possam acomodar de maneira mais refinada a heterogeneidade das empresas pertencentes à amostra, de forma a permitir a descrição mais detalhada das variáveis. Em termos gerenciais, espera-se que estes resultados possam balizar novos insights para os gestores analisarem as decisões de financiamento e investimento das empresas brasileiras.

\section{REVISÃO DE LITERATURA}

Do ponto de vista teórico, a Teoria de Trade-Off e a Teoria de Pecking Order são os dois modelos amplamente utilizados para analisar o comportamento de financiamento seguido pelas empresas. De acordo com a Teoria de Trade-Off, empresas rentáveis deveriam usar maior proporção de dívidas, na medida em que elas se beneficiariam dos proveitos fiscais. Por outro lado, quando existe o aumento do risco com a maior participação de capital de terceiros na estrutura da empresa, os acionistas tentem a exigir maior retorno de seu capital investido (Modigliani, \& Miller, 1963). Nesse sentido, Rajan e Zingales (1995) sugerem que a Teoria de Trade-Off prevê que as empresas mais rentáveis tenham uma relação positiva com o endividamento.

A Teoria de Pecking Order, por sua vez, sugere a existência de uma hierarquia de fontes de financiamento. Onde, primeiramente, seriam utilizados os recursos gerados de forma interna, em seguida, seriam utilizados recursos de terceiros, via endividamento, e a última opção seria a emissão de novas ações (Myers, 1984; Myers, \& Majluf, 1984). Portanto, espera-se que empresas com maior rentabilidade tenham maiores condições de se autofinanciar e, portanto, recorram menos ao uso de dívidas. Devido a isso, empresas com alto nível de alavancagem tendem ser menos rentáveis, do que empresas com menor grau de alavancagem.

Contudo, aumentar o endividamento pode em alguns casos não ser a melhor alternativa. Quando a empresa se encontra em uma posição de alta alavancagem, um incremento em seu endividamento pode aumentar, concomitantemente, o risco de insolvência. Como sugerido por Altman (1984) e Andrade e Kaplan (1998) um alto grau de alavancagem pode aumentar o risco de inadimplência e consequentemente o custo da dívida que, por sua vez, pode agravar o efeito negativo 
sobre a rentabilidade. Devido a esse conjunto de situações, a Teoria de Pecking Order sugere uma relação negativa entre a alavancagem e rentabilidade.

Existem, portanto, divergências teóricas entre as conjecturas emanadas pela Teoria de TradeOff e Teoria de Pecking Order. Os estudos empíricos também encontram divergências nessa relação. A literatura que analisa a relação entre alavancagem e rentabilidade, é vasta, entretanto, é preciso considerar que maioria das pesquisas atingiram conclusões ambíguas, ou contraditórias. Por um lado, diversos trabalhos encontraram uma relação positiva entre as variáveis. Por outro lado, um número considerável de estudos identificou uma relação negativa. E ainda, outros produziram resultados mistos, ou não encontraram uma relação estatisticamente significativa entre as variáveis.

De acordo com Berger e Bonaccorsi di Patti (2006) e Kyerboach-Coleman (2007), os ganhos decorrentes da alavancagem são significativos e podem aumentar o valor das empresas e sua rentabilidade. Utilizando dados de 2001 a 2010, Mantovani e Santos (2015) também descobriram que a alavancagem exerce um efeito positivo sobre a rentabilidade dos Bancos brasileiros listados na BM\&FBovespa. Tais considerações apontam que a alavancagem exerce um efeito positivo sobre a rentabilidade. Além destes, uma série de estudos fornecem evidências empíricas que apoiam a relação positiva entre essas variáveis (Roden, \& Lewellen, 1995; Hadlock, \& James, 2002; Ruland, \& Zhou, 2005; Rehman, 2013; Tsuruta, 2017).

Em contraste com a visão acima, outros estudos encontraram uma relação negativa entre alavancagem e rentabilidade. Negash (2001) descobriu que a dívida tem um impacto negativo sobre a rentabilidade das empresas listadas na Bolsa de Valores de Joanesburgo. Hussain, Shahid e Akmal (2016), por sua vez, identificaram que essa relação também ocorre nas empresas têxteis do Paquistão listadas na Bolsa de Valores de Karachi. Pinheiro, Reis e Avelino (2012) ao analisarem as empresas do setor de energia elétrica listadas na BM\&FBOVESPA, constataram que a dívida exerce um efeito negativo na rentabilidade dessas empresas. Além destes, outros estudos que relataram uma relação negativa entre as variáveis incluem os trabalhos de Michaelas, Chittenden e Poutzioris (1999) e Gedajlovic, Shapiro e Buduru (2003).

Outros trabalhos não encontram uma relação estatisticamente significativa (Amjed, 2007; Enekwe, Agu, \& Eziedo, 2014; Raheel, \& Shah, 2015; Alghusin, 2015). Portanto, nestes casos, o nível de alavancagem de uma empresa não exerce nenhum tipo de efeito sobre a sua capacidade de ganho. Outros exemplos de tal relação, são os trabalhos de Friend e Lang (1988), Bos e Fetherston (1993), Booth et al. (2001) e Abor (2005), que produziram resultados mistos.

Embora os trabalhos mencionados, apontem para diversos resultados, eles compartilham o objetivo de entender a relação entre alavancagem e rentabilidade. Tais considerações indicam que é imperativo ampliar a compreensão da relação entre essas variáveis. Considerando as evidências anteriores e apoiados pelo problema de pesquisa a seguinte hipótese é formulada:

H1: Existe uma relação estatisticamente significante entre alavancagem financeira e rentabilidade nas empresas brasileiras de capital aberto.

Embora existam evidências que sustentem uma relação entre alavancagem e rentabilidade, os trabalhos citados até então, presumem apenas uma relação linear entre as variáveis. Sabe-se por outro lado, que uma relação não linear é mais adequada para explicar a relação entre indicadores financeiros (Ragab, 2004). Como sugerido por Fattouh, Harris e Scaramozzino (2008) os modelos não lineares podem fornecer novas evidências sobre a estrutura de capital das empresas, especialmente quando observada a partir do nível de alavancagem, já que o efeito de uma variável sobre outra poderá ser diferente de acordo com a posição da empresa na distribuição de alavancagem. Tais considerações apontam que empresas em diferentes níveis de alavancagem têm diferentes graus de sensibilidade às mudanças das variáveis explicativas.

Ao levar em consideração essa perspectiva, é preciso considerar que pouco trabalho foi desenvolvido sob uma ótica não linear, algumas exceções são os trabalhos de Cheng, Liu e Chien 
(2010), Cuong e Canh (2012), Lin e Chang (2015), e Jaisinghani e Kanjilal (2017). Cheng, Liu e Chien (2010) encontraram três níveis limítrofes na dinâmica entre o nível de alavancagem e o valor da empresa nas companhias chinesas, sugerindo que dentro de uma amostra há três grupos distintos e menos heterogêneos do que a amostra inicial, nos quais uma variável exerce um efeito diferente sobre a outra. Cuong e Canh (2012), Lin e Chang (2015), e Jaisinghani e Kanjilal (2017), de forma semelhante, também segmentaram suas amostras em duas sub amostras menos heterogêneas com um relacionamento estrutural específico entre as variáveis, onde existem efeitos diferentes da alavancagem nas companhias do Vietnã, Taiwan, e Índia respectivamente.

Outro fator relevante nesse contexto, são as variáveis que são conjuntamente analisadas na relação entre alavancagem e rentabilidade. Embora um conjunto bastante amplo de indicadores tenham sido utilizado em modelos lineares, os não lineares, por sua vez, empregam duas principais variáveis como controle. Como verificado nos trabalhos anteriores (Cheng, Liu, \& Chien, 2010; Cuong, \& Canh, 2012; Lin, \& Chang, 2015; Jaisinghani, \& Kanjilal, 2017) o tamanho e o crescimento da empresa, têm sido empregados na análise entre alavancagem e rentabilidade.

De acordo com Berger e Bonaccorsi di Patti (2006) o tamanho de uma empresa exerce um efeito positivo sobre sua rentabilidade, isso, porque grandes empresas são geralmente mais diversificadas e possuem melhor gerenciamento. Além desses, há outros aspectos relevantes, como a capacidade de compensar perdas aleatórias, a capacidade tecnológica superior, os melhores recursos organizacionais (Onder, 2003; Tran, 2005), e a maior tolerância ao risco (Capon, Farley, \& Hoenig, 2011). Em virtude de tais características, espera-se que empresas de maior tamanho, tenham capacidade superior de atingir seus objetivos, e por consequência tenham maior rentabilidade. Sabese por outro lado, que em diversas situações ocorre o inverso. Um exemplo claro é encontrado nos trabalhos de Lin e Chang (2010) e Cheng, Liu e Chien (2010) que encontram evidências de uma relação negativa entre o tamanho e a rentabilidade. De acordo com estes pressupostos, o tamanho pode ter uma relação positiva ou negativa com a rentabilidade, devido a essa dualidade, têm-se a seguinte hipótese:

H2: Existe uma relação estatisticamente significante entre o tamanho e a rentabilidade das empresas brasileiras de capital aberto.

O crescimento também é considerado um fator relacionado a rentabilidade das empresas, uma vez que está associado ao aumento da produtividade, e ao crescimento de vendas, que tendem a afetar positivamente a rentabilidade (Mendelson, 2000; Cowling, 2004; Serrasquerio, Nunes, \& Sequeira, 2007; Jan, \& Park, 2011). Por outro lado, Greiner (1972) indica que um crescimento excessivo pode contribuir para a quebra das relações e das estruturas organizacionais estabelecidas ao longo do tempo, causando aumentos na formalidade e lentidão nos processos, que tendem a afetar negativamente a rentabilidade. Além deste, outros trabalhos relatam um efeito negativo do crescimento sobre a rentabilidade (Roper, 1999; Gschwandtner, 2005; Nakano, \& Kim, 2011). De acordo com estes pressupostos, é evidente que o crescimento possa ter uma relação positiva ou negativa com a rentabilidade, devido a essa dualidade, têm-se a seguinte hipótese:

H3: Existe uma relação estatisticamente significante entre o crescimento e a rentabilidade das empresas brasileiras de capital aberto.

Para analisar a relação entre alavancagem e rentabilidade nas empresas brasileiras, sob uma ótica não linear, foram delineados os procedimentos metodológicos a seguir. 


\section{MÉTODO}

A amostra foi constituída por empresas registradas como sociedades anônimas de capital aberto listadas na B3 S.A - Brasil, Bolsa, Balcão no período de 2009 a 2015, excluindo as empresas pertencentes ao setor financeiro devido às especificidades das informações contábeis desse setor que poderiam enviesar os resultados desta pesquisa. Neste período foram excluídos os dados de 2009 uma vez que esse exercício foi utilizado apenas para o cálculo de uma variável defasada. Os dados foram coletados na base de dados Economática e a matriz foi composta de dados balanceados, ou seja, foram excluídas as empresas com dados inexistentes para todo o período. A amostra final foi composta por 75 empresas, que representaram um total de 1650 observações.

Para operacionalização do modelo econométrico foi utilizada como variável dependente a rentabilidade, e como variável independente a alavancagem. Além dessas foram incluídas no modelo duas variáveis de controle: i) tamanho e ii) crescimento. A escolha destes indicadores foi amparada em critérios teóricos e aplicações empíricas em pesquisas anteriores, como discutido na seção 2. A definição das variáveis bem como as respectivas referências seguem descritas na Tabela 1.

Tabela 1 - Definição das variáveis

\begin{tabular}{|c|c|c|c|}
\hline \multicolumn{3}{|c|}{ Variável dependente } & \multirow{3}{*}{$\begin{array}{c}\text { Medida } \\
R O A=\frac{E B I T D A}{A T}\end{array}$} \\
\hline \multirow{2}{*}{\multicolumn{2}{|c|}{ Rentabilidade }} & & \\
\hline & & & \\
\hline $\begin{array}{l}\text { Variável } \\
\text { independente }\end{array}$ & Medida & $\begin{array}{c}\text { Sinal } \\
\text { Esperado }\end{array}$ & Autores \\
\hline \multirow{3}{*}{ Alavancagem } & \multirow{3}{*}{$A L A V=\frac{P C+P N C}{A T}$} & $(+)$ & $\begin{array}{l}\text { Roden e Lewellen (1995), Hadlock e James } \\
\text { (2002), Ruland e Zhou (2005), Berger e } \\
\text { Bonaccorsi di Patti (2006), Kyerboach-Coleman } \\
\text { (2007), Rehman (2013), Mantovani e Santos } \\
\text { (2015) e Tsuruta (2017). }\end{array}$ \\
\hline & & $(-)$ & $\begin{array}{l}\text { Rajan e Zingales (1995), Michaelas, Chittenden e } \\
\text { Poutzioris (1999), Negash (2001), Gedajlovic, } \\
\text { Shapiro e Buduru (2003), Pinheiro, Reis e } \\
\text { Avelino (2012) e Hussain, Shahid e Akmal } \\
\text { (2016) }\end{array}$ \\
\hline & & $\mathrm{n} / \mathrm{s}$ & $\begin{array}{l}\text { Amjed (2007), Enekwe, Agu e Eziedo (2014), } \\
\text { Raheel e Shah (2015), Alghusin (2015) }\end{array}$ \\
\hline $\begin{array}{l}\text { Variáveis de } \\
\text { controle }\end{array}$ & Medida & $\begin{array}{c}\text { Sinal } \\
\text { Esperado }\end{array}$ & Autores \\
\hline \multirow[t]{2}{*}{ Crescimento } & \multirow{2}{*}{$C R E=\frac{R E C_{i t}-R E C_{i t-1}}{R E C_{i t-1}}$} & $(+)$ & $\begin{array}{l}\text { Mendelson (2000), Cowling (2004), } \\
\text { Serrasquerio, Nunes e Sequeira (2007), e Jan e } \\
\text { Park (2011) }\end{array}$ \\
\hline & & $(-)$ & $\begin{array}{l}\text { Greiner (1972), Roper (1999), Gschwandtner } \\
\text { (2005) e Nakano e Kim (2011) }\end{array}$ \\
\hline \multirow[t]{2}{*}{ Tamanho } & \multirow[t]{2}{*}{$T A M_{i t}=\ln ^{*}(A T i t)$} & $(+)$ & $\begin{array}{l}\text { Berger e Bonaccorsi di Patti (2006), Onder } \\
\text { (2003), Tran (2005) e Capon, Farley e Hoenig } \\
\text { (2011) }\end{array}$ \\
\hline & & $(-)$ & Lin e Chang (2010) e Cheng, Liu e Chien (2010) \\
\hline Threshold: & Alavancagem financeira & & \\
\hline
\end{tabular}

Nota: Alavancagem (ALAV) é o passivo circulante (PC) mais o passivo não circulante (PNC), dividido pelo ativo total (AT); Rentabilidade (ROA) é o Lucros antes de juros, impostos, depreciação e amortização (EBITDA), dividido pelo ativo total (AT); Crescimento (CRE) é a variação da receita de vendas liquida (REC) das empresas entre os anos $t$ - 1 e t; Tamanho (TAM) que é o logaritmo natural do ativo total (AT); A variável threshold é a Alavancagem.

Fonte: Elaborado pelos autores (2018)

Os dados foram analisados através do software R e ocorreram em duas etapas. A primeira foi o cálculo das estatísticas descritivas nas variáveis, e a segunda, baseada na metodologia proposta por Hansen (1999), foi a aplicação de dados em painel com threshold.

Este modelo permite identificar e descrever os saltos ou quebras estruturais nas variáveis de um modelo econométrico, segmentando a amostra com base em uma variável determinada. Sendo 
assim, uma amostra inicialmente heterogênea pode ser segmentada em duas, três ou quatro sub amostras menos heterogêneas onde a sensibilidade da variável dependente às covariáveis pode mudar entre os regimes. Dessa forma, pode-se obter um relacionamento estrutural específico entre as variáveis.

O modelo proposto por Hansen (1999) permite que o coeficiente da equação de regressão possa mudar de valor dependendo da sub amostra ou regime em que se encontra. O modelo com dois regimes (single-threshold) pode ser especificado da seguinte forma:

$$
y_{i t}=\mu_{i}+x_{i t} I\left(q_{i t} \leq \gamma\right) \beta_{1}+x_{i t} I\left(q_{i t}>\gamma\right) \beta_{2}+\varepsilon_{i t}
$$

Em (1) $I$ é uma função indicadora assumindo valores de $I=1$, quando $\left(q_{i t} \leq \gamma\right)$ e 0 nos outros casos, e $I=1$, quando $\left(q_{i t}>\gamma\right)$ e 0 nos outros casos; $q_{i t}$ é a variável threshold, $\gamma$ o parâmetro threshold que divide a equação em dois regimes com coeficientes $\beta=\left(\beta_{1}, \beta_{2}\right)$; $\varepsilon_{i t}$ é o termo de erro assumido ser independente e identicamente distribuído (iid), podendo ser heterocedástico.

Para cada valor de $\gamma \in \Gamma$, o vetor $z_{i t}(\gamma)$ assume uma forma específica. A estimação dos coeficientes é através de OLS e a seleção é por grid search das estimativas dos coeficientes que gerem menor Sum of Squared Error (SSE), ou seja, para cada valor de $\gamma \in \Gamma$, obtêm-se por OLS os coeficientes e a Sum of Squared Error $\left(S S E_{\gamma}=\sum \sum \varepsilon_{i t}^{2}\right)$. As estimativas mais adequadas são as que minimizam a função $S S E_{\gamma}$ no espaço $\Gamma$.

O modelo com três regimes (double-threshold) pode ser descrito como (2).

$$
y_{i t}=\mu_{i}+x_{i t} I\left(q_{i t} \leq \gamma_{1}\right) \beta_{1}+x_{i t} I\left(\gamma_{1}<q_{i t} \leq \gamma_{2}\right) \beta_{2}+x_{i t} I\left(\gamma_{2}<q_{i t}\right) \beta_{3}+\varepsilon_{i t}
$$

Uma forma alternativa de escrever o modelo double-threshold é especificada em (3).

$$
y_{i t}=\left\{\begin{array}{lr}
\mu_{i}+\beta_{1} x_{i t}+\varepsilon_{i t}, & q_{i t} \leq \gamma_{1}, \\
\mu_{i}+\beta_{2} x_{i t}+\varepsilon_{i t}, & \gamma_{1}<q_{i t} \leq \gamma_{2}, \\
\mu_{i}+\beta_{3} x_{i t}+\varepsilon_{i t}, & \gamma_{2}<q_{i t} .
\end{array}\right.
$$

Em (3), a amostra é dividida em três regimes dependendo apenas se a variável threshold é menor, maior ou está entre um intervalo de valores definidos pelos thresholds. Esse procedimento garante maior homogeneidade dentro de cada regime, que facilita a obtenção de coeficientes mais realistas. O modelo de Hansen (1999) suporta até três thresholds. Para uma melhor compreensão do processo de estimação, é considerada uma representação alternativa das equações (2) e (3), dado por (4).

$$
y_{i t}=\mu_{i}+\beta z_{i t}\left(\gamma_{1}, \gamma_{2}\right)+\varepsilon_{i}
$$

Em (4), $z_{i t}\left(\gamma_{1}, \gamma_{2}\right)=\left(x_{i t} I\left(q_{i t} \leq \gamma_{1}\right), x_{i t} I\left(\gamma_{1}<q_{i t} \leq \gamma_{2}\right), x_{i t} I\left(\gamma_{2}<q_{i t}\right)\right)$ e $B=\left(\beta_{1}, \beta_{2}\right.$ e $\left.\beta_{3}\right)$. Observe que para cada par $\left(\gamma_{1}, \gamma_{2}\right) \in \Gamma \times \Gamma$, o vetor $z_{i t}=\left(\gamma_{1}, \gamma_{2}\right)$ assumirá uma forma específica. A estimação dos coeficientes é através de OLS e a seleção é por grid search das estimativas dos coeficientes que gerem menor Sum of Squared Error, ou seja, para cada valor de $\gamma_{1} \mathrm{e} \gamma_{2} \in \Gamma \times \Gamma$, obtêmse por OLS os coeficientes e a Sum of Squared Error $\left(S S E_{\gamma 1, \gamma 2}=\sum \sum \varepsilon_{i t}^{2}\left(\gamma_{1}, \gamma_{2}\right)\right)$, as estimativas mais adequadas são as que minimizam a função $S S E_{\gamma 1, \gamma 2}$.

Para valores de $\left(\gamma_{1}, \gamma_{2}\right)$ os coeficientes $\left(\beta_{1}, \beta_{2}\right.$ e $\left.\beta_{3}\right)$ são lineares e a estimação por OLS através do grid search é adequada. Os coeficientes são aqueles que minimizam a Sum of Squared Error $\left(S S E_{\gamma 1, \gamma_{2}}=\sum \sum \varepsilon_{i t}^{2}\left(\gamma_{1}, \gamma_{2}\right)\right)$. 
No contexto do modelo (1), é necessário verificar a significância do efeito threshold $(\gamma)$, ou seja, se a diferenca $\beta_{1}-\beta_{2}$ é suficientemente grande para que $(\gamma)$ seja significativo. Para tal, utilizase um teste de Multiplicador de Lagrange (LR) descrito em (5.a, 5.b e 5.c).

$$
\begin{gathered}
L R(\gamma)=(\operatorname{SSE}(\operatorname{lm})-\operatorname{SSE}(\gamma)) / \sigma_{\gamma}^{2} \\
\operatorname{LR}(\gamma 1, \gamma 2)=(\operatorname{SSE}(\gamma)-\operatorname{SSE}(\gamma 1, \gamma 2)) / \sigma_{\gamma 1, \gamma 2}^{2} \\
\operatorname{LR}(\gamma 1, \gamma 2, \gamma 3)=(\operatorname{SSE}(\gamma 1, \gamma 2)-\operatorname{SSE}(\gamma 1, \gamma 2, \gamma 3)) / \sigma_{\gamma 1, \gamma 2, \gamma 3}^{2}
\end{gathered}
$$

O teste LR é robusto à heterocedasticidade e tem seus valores críticos determinados por um procedimento boostrap. Em (5.a), se o valor da estatística LR superar o valor crítico, conclui-se que existem dois regimes, onde a associação entre a variável dependente e as variáveis independentes é distinta, pelo menos para uma das variáveis. Por outro lado, se a estatística LR não superar o valor crítico, conclui-se que o modelo linear $(\mathrm{lm})$ de efeito fixo é o mais adequado. A análise para 5.b e 5.c é semelhante à 5.a, porém a comparação realizada é 1vs2 threshold e 2 vs3 threshold.

\section{ANÁLISE DOS RESULTADOS}

Inicialmente na Tabela 2 são apresentadas as estatísticas descritivas das quatro variáveis analisadas. Os resultados mostram que as variáveis rentabilidade e tamanho têm médias e medianas muito próximas, o que não se verifica no caso da alavancagem em que a mediana é levemente superior à média, e no crescimento em que a média é levemente superior a mediana. As variáveis com maior dispersão foram: (i) crescimento, (ii) alavancagem e (iii) tamanho, confirmadas pela diferença entre o mínimo e o máximo. Pode-se observar uma forte oscilação dos valores de crescimento, embora esta variável tenha apresentado média de $(0,057)$ os valores de mínimo e máximo, indicam que as empresas no período tiveram um crescimento negativo $(-0,923)$ e um elevado crescimento positivo de $(10,562)$.

Os valores associados à assimetria indicam que todas as variáveis possuem uma distribuição assimétrica, algumas concentradas à esquerda, e outras à direita da média. Já os valores associados à curtose, indicam que todas as variáveis têm uma distribuição leptocúrtica. Também se observa a partir do desvio padrão que a variável de alavancagem foi a que mais oscilou $(0,975)$, seguida pelo crescimento $(0,526)$ e tamanho $(0,389)$. A variável de rentabilidade apresentou o menor desvio padrão $(0,036)$.

Tabela 2 - Estatística descritiva das variáveis das 75 empresas (período de março/2010 a dezembro/2015, dados trimestrais).

\begin{tabular}{lcccc}
\hline & Alavancagem & Crescimento & Rentabilidade & Tamanho \\
\hline Observações & 1.650 & 1.650 & 1.650 & 1.650 \\
Mínimo & 0,000 & $-0,923$ & $-0,682$ & 0,000 \\
Máximo & 5,773 & 10,562 & 0,226 & 2,073 \\
1. Quartil & 3,717 & $-0,068$ & 0,008 & 1,604 \\
3. Quartil & 4,210 & 0,108 & 0,033 & 1,796 \\
Média & 3,796 & 0,057 & 0,021 & 1,624 \\
Mediana & 4,006 & 0,015 & 0,020 & 1,695 \\
SE Média & 0,024 & 0,013 & 0,001 & 0,010 \\
LCL Média & 3,749 & 0,032 & 0,020 & 1,605 \\
UCL Média & 3,843 & 0,082 & 0,023 & 1,642 \\
Variância & 0,951 & 0,277 & 0,001 & 0,152 \\
Desvio padrão & 0,975 & 0,526 & 0,036 & 0,389 \\
Assimetria & $-2,594$ & 12,268 & $-5,765$ & $-3,246$ \\
Curtose & 7,833 & 194,752 & 104,910 & 10,900 \\
\hline
\end{tabular}

Fonte: Elaborado pelos autores (2018)

Posteriormente, em uma segunda etapa de análise, foi empregado o modelo de dados em painel com threshold proposto por Hansen (1999). De acordo com este método uma amostra 
inicialmente homogênea pode ser dividida em classes ou regimes estabelecidos endogenamente e com potencial de acomodar adequadamente a heterogeneidade dos indivíduos na amostra. Para tal, é necessário estabelecer uma variável como threshold do modelo. Devido às evidências de que o efeito de uma variável sobre outra poderá ser diferente de acordo com a posição da empresa na distribuição de alavancagem (Fattouh, Harris, \& Scaramozzino, 2008), esta variável foi definida como o threshold do modelo.

Para verificar a existência do efeito threshold é testada a hipótese nula de um modelo linear contra a hipótese alternativa de um modelo com efeito threshold. Este teste foi realizado sequencialmente para zero, um, dois ou três efeitos. Através do procedimento de bootstrap repetido 500 vezes para cada um dos três testes de limite do painel foram obtidas as estatísticas do teste LR juntamente com seus p-valores, de acordo com a Tabela 3.

Tabela 3 - Teste LR para determinação do número de thresholds.

\begin{tabular}{|c|c|c|c|c|c|c|c|}
\hline \multirow[t]{2}{*}{ Modelo } & \multirow{2}{*}{ SSE } & \multicolumn{3}{|c|}{ Threshold } & \multirow{2}{*}{$\begin{array}{c}\text { LR } \\
\text { Test }\end{array}$} & \multirow{2}{*}{$\begin{array}{c}\text { Valor Crítico } \\
(5 \%)\end{array}$} & \multirow{2}{*}{ Sig. } \\
\hline & & 1 & 2 & 3 & & & \\
\hline Zero Threshold & 1,263 & & & & & & \\
\hline Único Threshold & 1,233 & 4,106 & & & 38,008 & 32,711 & $<0,05$ \\
\hline Duplo Threshold & 1,225 & 4,106 & 4,407 & & 9,775 & 27,483 & $>0,10$ \\
\hline Triplo Threshold & 1,223 & 4,106 & 4,407 & 4,732 & 2,243 & 28,698 & $>0,10$ \\
\hline
\end{tabular}

Fonte: elaborado pelos autores (2018)

O Teste LR para efeito único do threshold foi significativo pois o valor do Teste LR $(38,008)$ é superior ao valor crítico de 5\% e apresenta redução do valor da soma dos quadrados dos erros (SSE) em relação ao teste com zero threshold. O Teste LR verifica o efeito threshold e observado o valor deste teste conclui-se em favor de utilizar um modelo com único efeito threshold $(\mathrm{LR}=38,008)$. Isso indica que para analisar a relação entre alavancagem e rentabilidade, o modelo que considera uma relação não linear é o mais adequado.

Destaca-se que o modelo ideal apresenta uma menor SSE e quanto menor, mais ajustado o modelo está. Desta maneira, é possível verificar que quando aumenta o número de thresholds o SSE diminuí, isso indica que o modelo fica mais ajustado e melhor explica a relação entre as variáveis. O efeito duplo e triplo threshold apesar de apresentarem uma SSE inferior ao efeito único a estatística do Teste LR não supera o valor crítico, portanto, não são considerados como o modelo que melhor explica a relação entre as variáveis.

Com base nessa interpretação, verifica-se que a amostra é dividida em dois grupos. No primeiro regime, estão as empresas com menor alavancagem (alav $\leq 4,106$ ), e no segundo regime estão as empresas com maior alavancagem (alav > 4,106). Depois de identificar o número de regimes, são apresentados na Tabela 4 os resultados para o modelo de dados em painel com threshold considerando os dois regimes.

Tabela 4 - Resultado para o modelo de dados em painel com threshold (período de março/2010 até dezembro/2015, dados trimestrais).

\begin{tabular}{lrrr}
\hline Variável dependente & \multicolumn{2}{c}{ Variáveis independentes } \\
\hline Rentabilidade & Alavancagem & Tamanho & Crescimento \\
\hline $1^{\mathbf{0}}$ Regime (Alavancagem $\left.\leq 4,106\right)$ & $-0,006$ & $\mathbf{0 , 0 3 1}$ & 0,001 \\
& $(-1,306)$ & $(3,011)$ & $(1,309)$ \\
$2^{\mathbf{o}}$ Regime (Alavancagem $>$ 4,106) & $\mathbf{- 0 , 0 3 5}$ & $\mathbf{0 , 0 9 9}$ & 0,001 \\
& $(-3,878)$ & $(4,439)$ & $(1,309)$ \\
\hline
\end{tabular}

A linha principal é a estimativa do coeficiente, e o teste $t$ está entre parênteses.

Fonte: elaborado pelos autores (2018)

Ao observar os coeficientes estimados é possível verificar que no primeiro regime, onde estão as empresas com menor grau de alavancagem (alav $\leq 4,106)$, não existe uma relação significativa entre alavancagem financeira e rentabilidade, ou seja, a presença de dívidas na estrutura de capital 
das empresas não está associada à rentabilidade destas empresas. Evidências semelhantes já foram documentadas anteriormente (Amjed, 2007; Enekwe, Agu, \& Eziedo, 2014; Raheel, \& Shah, 2015; Alghusin, 2015). Já no segundo regime, onde estão as empresas com maior nível de alavancagem (alav >4,106) as variáveis possuem uma relação significativa e negativa. Ou seja, um alto nível alavancagem exerce um efeito negativo sobre a rentabilidade, corroborando com os achados de Rajan e Zingales (1995), Michaelas, Chittenden e Poutzioris (1999), Negash (2001), Gedajlovic, Shapiro e Buduru (2003), Pinheiro, Reis e Avelino (2012) e Hussain, Shahid e Akmal (2016).

Uma possível explicação para a relação negativa nas empresas com maior alavancagem pode ser devido à bem conhecida hierarquia entre as fontes de financiamento sugerida pela Teoria de Pecking Order (Myers, 1984, Myers, \& Majluf, 1984). De acordo com esta perspectiva, empresas menos rentáveis tendem a ter um alto nível de alavancagem, uma vez que apenas as empresas rentáveis têm a oportunidade de financiar novos investimentos com lucros acumulados e não com uso de dívidas ou com a emissões de ações. Outro aspecto relevante pode estar associado ao custo da dívida. Como sugerido por Altman (1984) e Andrade e Kaplan (1998) um alto grau de alavancagem pode aumentar o risco de insolvência e consequentemente o custo da dívida, o que pode agravar o efeito negativo sobre a rentabilidade.

A variável tamanho apresenta um coeficiente positivo e significativo de $(0,031)$ e $(0,099)$ no primeiro e segundo regime, respectivamente. Esse resultado indica que independentemente do nível de alavancagem o tamanho é uma variável determinante em sua rentabilidade. Esse resultado corrobora com os achados de Berger e Bonaccorsi di Patti (2006), Onder (2003), Tran (2005) e Capon et al. (2011) que justificam um efeito positivo devido a um conjunto de características tais como: i) diversificação, ii) melhor gerenciamento, iii) capacidade de compensar perdas aleatórias, iv) capacidade tecnológica superior, v) melhores recursos organizacionais, e vi) maior tolerância ao risco. De acordo com os autores, essas características ou o conjunto delas favorece as empresas de maior tamanho em sua capacidade de atingir seus objetivos, e por consequência tenham maior rentabilidade.

A variável crescimento, no primeiro assim como no segundo regime não possui uma relação significativa com a rentabilidade, em outras palavras, independentemente do nível de alavancagem o crescimento não exerce nenhum efeito sobre a rentabilidade das empresas. Esse resultado contraria diversos trabalhos anteriores (Greiner, 1972; Roper, 1999; Mendelson, 2000; Cowling, 2004; Gschwandtner, 2005; Serrasquerio et al. 2007; Jan, \& Park, 2011; Nakano, \& Kim, 2011) que embora apresentem resultados opostos quanto ao efeito, indicam a existência de uma relação significativa. Isso implica que a expansão da taxa de crescimento não aumenta necessariamente a rentabilidade das empresas brasileiras, seja qual for seu nível de alavancagem.

Na Tabela 5, são sintetizados os resultados de acordo com os dois regimes encontrados nesse estudo.

Tabela 5 - Relação teórica esperada e relação encontrada entre rentabilidade e as variáveis independentes.

\begin{tabular}{lccc}
\multicolumn{2}{l}{ Tabela 5 - Relação teórica esperada e relação encontrada entre rentabilidade e as variáveis independentes. } \\
\hline \multirow{2}{*}{ Alavancagem } & Sinal Esperado & Regime & Relação Encontrada \\
\hline \multirow{2}{*}{ Crescimento } & $(+) /(-) /(\mathrm{n} / \mathrm{s})$ & $1^{\circ}$ & $\mathrm{n} / \mathrm{s}$ \\
& $(+) /(-)$ & $2^{\mathrm{o}}$ & $(-)$ \\
\hline \multirow{2}{*}{ Tamanho } & $(+) /(-)$ & $1^{\circ}$ & $\mathrm{n} / \mathrm{s}$ \\
& & $2^{\mathrm{o}}$ & $\mathrm{n} / \mathrm{s}$ \\
\hline
\end{tabular}

Fonte: elaborado pelos autores (2018)

\section{CONSIDERAÇÕES FINAIS}

Este trabalho analisou a relação não linear entre alavancagem financeira e rentabilidade nas empresas brasileiras de capital aberto. Para tal, desenvolveu-se uma pesquisa em uma amostra composta por 75 companhias brasileiras listadas na B3 com dados disponíveis no período de 2009 a 2015. Amparado em um modelo de dados em painel com threshold foi traçado detalhadamente a 
distribuição de alavancagem das empresas brasileiras, mostrando à heterogeneidade condicional da rentabilidade às suas variáveis explicativas. Coeficientes que antes eram vistos como homogêneos entre as empresas, agora foram analisados e distinguidos por nível de alavancagem, manifestando entre si diferenças estatisticamente significantes. Até então, esse ponto era desconsiderado nos estudos brasileiros que examinavam de maneira linear a relação da alavancagem financeira com a rentabilidade das empresas.

Os resultados demostram que as variáveis explicativas podem ter comportamentos diferenciados entre os regimes. Por meio de uma abordagem não linear é evidenciado que nas empresas com menor nível de alavancagem a presença de dívidas na estrutura de capital não está estatisticamente relacionada à sua rentabilidade. Todavia, nas empresas com níveis elevados de alavancagem existe uma relação significativa e negativa entre alavancagem e rentabilidade. Esse resultado permite aceitar a hipótese $\mathrm{H} 1$ de existência de uma relação apenas para as empresas com maior nível de alavancagem.

Adicionalmente, investigou-se o impacto das variáveis tamanho e crescimento sobre a rentabilidade. A variável tamanho no primeiro, assim como no segundo regime, possui uma relação significativa e positiva com a rentabilidade. Ou seja, independentemente do nível de alavancagem, o tamanho exerce um efeito positivo sobre a rentabilidade. Este resultado permite aceitar a hipótese H2, de existência de uma relação significativa em todas as empresas da amostra. Quanto à variável crescimento, esperava-se confirmar a existência de uma relação significativa entre as variáveis, entretanto, constatou-se que não existe uma relação significativa em nenhum dos regimes. Portanto, o crescimento não exerce nenhum efeito sobre a rentabilidade das empresas brasileiras. Esse resultado não permite aceitar a hipótese H3.

As conclusões gerais desta pesquisa são favoráveis a Teoria de Pecking Order (Myers, 1984; Myer, \& Majluf, 1984) e recomendam a existência uma relação não linear entre alavancagem e rentabilidade nas empresas brasileiras de capital aberto. Esse resultado confirma empiricamente que a rentabilidade das empresas brasileiras é afetada de formas distintas dependendo do seu nível de alavancagem como já destacado por Fattouh, Harris e Scaramozzino (2008). Esse resultado é consistente com os trabalhos de Cheng, Liu e Chien (2010), Cuong e Canh (2012), Lin e Chang (2015), e Jaisinghani e Kanjilal (2017) que de uma forma semelhante também indicaram a presença de dinâmicas não lineares na estrutura de capital das empresas.

Estes resultados contribuem com a difusão do conhecimento sobre o assunto e apresentam novas evidências sobre a relação entre alavancagem e rentabilidade nas empresas brasileiras. A descoberta de que empresas mais alavancadas são afetadas negativamente em sua rentabilidade trazem algumas implicações úteis para os gestores das empresas. As decisões de orçamento de capital são baseadas principalmente na comparação entre o custo de capital e os retornos esperados. As evidências apresentadas neste estudo demonstram que além destes parâmetros os gestores também podem considerar o nível de alavancagem ao tomar as decisões de orçamento de capital. Analistas e investidores também devem considerar o nível de alavancagem na avaliação de empresas.

Importante observar que esse estudo apresenta algumas limitações. $\mathrm{O}$ modelo de dados em painel com threshold possui limitações ao uso de variáveis dummy o que impossibilita a análise do efeito setorial. Além disso, um modelo deste tipo não comporta um número elevado de varáveis explicativas. Obviamente, essa limitação é um estímulo ao desenvolvimento de novas pesquisas. Trabalhos futuros poderiam incluir outras variáveis que possam explicar a rentabilidade das empresas brasileiras. Nesse sentido, o procedimento metodológico adotado neste trabalho pode ser reproduzido com outras variáveis fornecendo novas evidências sobre os fatores que podem explicar a rentabilidade das empresas.

\section{REFERÊNCIAS}

Abor, J. (2005). The effect of capital structure on profitability: an empirical analysis of listed firms in Ghana. The Journal of Risk Finance, 6(5), 438-445. 
Alghusin, N. A. S. (2015). Do Financial Leverage, Growth and Size Affect Profitability of Jordanian Industrial Firms Listed. International Journal of Academic Research in Business and Social Sciences, 5(4), 385-398.

Altman, E. I. (1984). A further empirical investigation of the bankruptcy cost question. The Journal of Finance, 39(4), 1067-1089.

Amjed, S. (2007). The impact of financial structure on profitability: Study of Pakistan's textile sector. Management of International Business and Economic Systems, 3(2), 440-450.

Andrade, G., \& Kaplan, S. N. (1998). How costly is financial (not economic) distress? Evidence from highly leveraged transactions that became distressed. The Journal of Finance, 53(5), 1443-1493.

Bastos, D. D., \& Nakamura, W. T. (2009). Determinantes da estrutura de capital das companhias abertas no Brasil, México e Chile no período 2001-2006. Revista Contabilidade \& Finanças, 20(50), 75-94.

Bastos, D. D., Nakamura, W. T., \& Basso, L. F. C. (2009). Determinantes da estrutura de capital das companhias abertas na América Latina: um estudo empírico considerando fatores macroeconômicos e institucionais. Revista de Administração Mackenzie, 10(6), 47-77.

Berger, A. N., \& Di Patti, E. B. (2006). Capital structure and firm performance: A new approach to testing agency theory and an application to the banking industry. Journal of Banking \& Finance, 30(4), 1065-1102.

Booth, L., Aivazian, V., Demirguc-Kunt, A., \& Maksimovic, V. (2001). Capital structures in developing countries. The Journal of Finance, 56(1), 87-130.

Bos, T., \& Fetherston, T. A. (1993). Capital structure practices on the specific firm. Research in International Business and Finance, 10(3), 53-66.

Bradley, M., Jarrell, G. A., \& Kim, E. H. (1984). On the existence of an optimal capital structure: Theory and evidence. The Journal of Finance, 39(3), 857-878.

Capon, N., Farley, J. U., \& Hoenig, S. (1990). Determinants of financial performance: a meta-analysis. Management Science, 36(10), 1143-1159.

Ceretta, P. S., Vieira, K. M., da Fonseca, J. L., \& de Lima Trindade, L. (2009). Determinantes da estrutura de capital: uma análise de dados em painel de empresas pertencentes ao Ibovespa no período de 1995 a 2007. Revista de Gestão, 16(4), 29-43.

Cheng, Y. S., Liu, Y. P., \& Chien, C. Y. (2010). Capital structure and firm value in China: A panel threshold regression analysis. African Journal of Business Management, 4(12), 2500-2507.

Correa, C. A., Basso, L. F. C., \& Nakamura, W. T. (2013). A estrutura de capital das maiores empresas brasileiras: análise empírica das teorias de pecking order e trade-off usando panel data. Revista de Administração Mackenzie, 14(4).

Cowling, M. (2004). The Growth-Profit Nexus. Small Business Economics, 22, 1-9.

Cuong, N. T., \& Canh, N. T. (2012). The effect of capital structure on firm value for Vietnam's seafood processing enterprises. International Research Journal of Finance and Economics, 89, 221-233.

DeAngelo, H., \& Roll, R. (2015). How stable are corporate capital structures? The Journal of Finance, 70(1), 373-418.

Devos, E., Dhillon, U., Jagannathan, M., \& Krishnamurthy, S. (2012). Why are firms unlevered? Journal of Corporate Finance, 18(3), 664-682.

Enekwe, C. I., Agu, C. I., \& Nnagbogu, E. K. (2014). The effect of financial leverage on financial performance: Evidence of quoted pharmaceutical companies in Nigeria. Journal of Economics and Finance, 5(3), 17-25.

Famá, R., \& Kayo, E. K. (1997). Teoria de agência e crescimento: evidências empíricas dos efeitos positivos e negativos do endividamento. Caderno de Pesquisas em Administração, 2(5), 1-8.

Fattouh, B., Harris, L., \& Scaramozzino, P. (2008). Non-linearity in the determinants of capital structure: evidence from UK firms. Empirical Economics, 34(3), 417-438.

Frank, M. Z., \& Goyal, V. K. (2009). Capital structure decisions: which factors are reliably important? Financial Management, 38(1), 1-37.

Friend, I., \& Lang, L. H. (1988). An empirical test of the impact of managerial self-interest on corporate capital structure. The Journal of Finance, 43(2), 271-281.

Gedajlovic, E., Shapiro, D. M., \& Buduru, B. (2003). Financial ownership, diversification and firm profitability in Japan. Journal of Management and Governance, 7(3), 315-335.

González, V. M. (2013). Leverage and corporate performance: International evidence. International Review of Economics \& Finance, 25, 169-184. 
Graham, J. R., Leary, M. T., \& Roberts, M. R. (2015). A century of capital structure: The leveraging of corporate America. Journal of Financial Economics, 118(3), 658-683.

Greiner, L. (1972). Evolutions and Revolutions as Organizations Grow. Harvard Business Review, 50, $37-$ 46.

Gschwandtner, A. (2005). Profit persistence in the 'very'long run: evidence from survivors and exiters. Applied Economics, 37(7), 793-806.

Hadlock, C. J., \& James, C. M. (2002). Do banks provide financial slack? The Journal of Finance, 57(3), 1383-1419.

Hansen, B. E. (1999). Threshold effects in non-dynamic panels: Estimation, testing, and inference. Journal of Econometrics, 93(2), 345-368.

Harris, M., \& Raviv, A. (1991). The theory of capital structure. The Journal of Finance, 46(1), 297-355.

Hussein, M., Shahid, H., \& Akmal, M. (2016). Effect of Profitability and Financial Leverage on Capita Structure in Pakistan Textile Firms. Arabian Journal of Business and Management Review, 6(4), 14.

Jaisinghani, D., \& Kanjilal, K. (2017). Non-linear dynamics of size, capital structure and profitability: Empirical evidence from Indian manufacturing sector. Asia Pacific Management Review, 22(3), 159165.

Jang, S. S., \& Park, K. (2011). Inter-relationship between firm growth and profitability. International Journal of Hospitality Management, 30(4), 1027-1035.

Kayo, E. K., \& Kimura, H. (2011). Hierarchical determinants of capital structure. Journal of Banking \& Finance, 35(2), 358-371.

Kyereboah-Coleman, A. (2007). The impact of capital structure on the performance of microfinance institutions. The Journal of Risk Finance, 8(1), 56-71.

Lang, L., Ofek, E., \& Stulz, R. (1996). Leverage, investment, and firm growth. Journal of Financial Economics, 40(1), 3-29.

Lin, F. L., \& Chang, T. (2010). Does family ownership affect firm value in Taiwan? A panel threshold regression analysis. International Research Journal of Finance and Economics, 42(1), 45-53.

Lin, F. L., \& Chang, T. (2011). Does debt affect firm value in Taiwan? A panel threshold regression analysis. Applied Economics, 43(1), 117-128.

Mantovani, M. H. C., \& dos Santos, J. O. (2015). Análise da relação entre alavancagem e rentabilidade dos bancos brasileiros listados na Bolsa de Valores de São Paulo no período de 2001 a 2010. Revista de Gestão, 22(4), 509-524.

Mendelson, H. (2000). Organizational architecture and success in the information technology industry. Management Science, 46(4), 513-529.

Michaelas, N., Chittenden, F., \& Poutziouris, P. (1999). Financial policy and capital structure choice in UK SMEs: Empirical evidence from company panel data. Small Business Economics, 12(2), 113-130.

Modigliani, F., \& Miller, M. H. (1958). The cost of capital, corporation finance and the theory of investment. The American Economic Review, 48(3), 261-297.

Modigliani, F., \& Miller, M. H. (1963). Corporate income taxes and the cost of capital: a correction. The American Economic Review, 53(3), 433-443.

Myers, S. C. (1977). Determinants of corporate borrowing. Journal of Financial Economics, 5(2), 147-175.

Myers, S. C. (1984). The capital structure puzzle. The Journal of Finance, 39(3), 574-592.

Myers, S. C., \& Majluf, N. S. (1984). Corporate financing and investment decisions when firms have information that investors do not have. Journal of Financial Economics, 13(2), 187-221.

Nakano, A., \& Kim, D. (2011). Dynamics of growth and profitability: The case of Japanese manufacturing firms. Global Economic Review, 40(1), 67-81.

Negash, M. (2001). Debt, tax shield and bankruptcy costs: Some evidence from JSE. Investment Analysts Journal, 30(54), 33-44.

Omran, M., \& Ragab, A. (2004). Linear versus non-linear relationships between financial ratios and stock returns: empirical evidence from Egyptian firms. Review of Accounting and Finance, 3(2), 84-102.

Onder, Z. Ownership concentration and Firm Performance: Evidence from Turkish firms. METU Studies in Development, 30(2), 181-203.

Perobelli, F. F. C., \& Famá, R. (2003). Fatores determinantes da estrutura de capital para empresas latinoamericanas. Revista de Administração Contemporânea, 7(1), 9-35. 
Pinheiro, L. E. T., Reis, D. E. A., \& Avelino, B. C. (2016). Análise da relação entre endividamento e rentabilidade em empresas do setor de energia elétrica listadas na BM\&FBOVESPA. Revista Mineira de Contabilidade, 3(47), 26-35.

Raheel, T., \& Shah, F. M. (2015). A Study That Identify the Relationship between the Financial Leverage and Firms Profitability: Empirical Evidence from Oil and Gas Companies of Pakistan Listed In KSE. International Journal of Scientific and Engineering Research, 6(11), 80-88.

Rajan, R. G., \& Zingales, L. (1995). What do we know about capital structure? Some evidence from international data. The Journal of Finance, 50(5), 1421-1460.

Rehman, S. S. F. U. (2013). Relationship between financial leverage and financial performance: Empirical evidence of listed sugar companies of Pakistan. Global Journal of Management and Business Research, 13(1), 33-40.

Roden, D. M., \& Lewellen, W. G. (1995). Corporate capital structure decisions: evidence from leveraged buyouts. Financial Management, 24, 76-87.

Roper, S. (1999). Modelling small business growth and profitability. Small Business Economics, 13(3), 235-252.

Ruland, W.; Zhou, P. (2005). Debt, diversification and valuation, Review of Quantitative Financial Accounting, 25(3), 277-291.

Serrasqueiro, Z., Macas Nunes, P., \& Tiago Neves Sequeira, S. (2007). Firms' growth opportunities and profitability: a nonlinear relationship. Applied Financial Economics Letters, 3(6), 373-379.

Silva, E. D. S., Santos, J. F. D., Perobelli, F. F. C., \& Nakamura, W. T. (2016). Capital Structure of Brazil, Russia, India and China by economic Crisis. Revista de Administração Mackenzie, 17(3), 105-131.

Titman, S., \& Wessels, R. The determinants of capital structure choice. The Journal of Finance, 43(1), 119.

Tran, G. (2005). Ownership structure and firm performance in transition countries: The case of European Union new members. In Anais Dans FMA European Conference.

Tsuruta, D. (2017). Variance of firm performance and leverage of small businesses. Journal of Small Business Management, 55(3), 404-429. 